\title{
Unsupervised inference approach to facial attractiveness
}

\author{
Miguel Ibanez-Berganza ${ }^{\text {Corresp., } 1}{ }^{,}$Ambra Amico ${ }^{2}$, Gian Luca Lancia ${ }^{1}$, Federico Maggiore ${ }^{1}$, Bernardo Monechi ${ }^{3}$, \\ Vittorio Loreto ${ }^{1,3,4}$ \\ ${ }^{1}$ Department of Physics, University of Roma "La Sapienza", Rome, Italy \\ 2 Chair of Systems Design, Swiss Federal Institute of Technology, Zurich, Zurich, Switzerland \\ 3 SONY Computer Science Laboratories, Paris, France \\ ${ }^{4}$ Complexity Science Hub, Vienna, Austria \\ Corresponding Author: Miguel Ibanez-Berganza \\ Email address: miguel.berganza@roma1.infn.it
}

The perception of facial attractiveness is a complex phenomenon which depends on how the observer perceives not only individual facial features, but also their mutual influence and interplay. In the machine learning community, this problem is typically tackled as a problem of regression of the subject-averaged rating assigned to natural faces. However, it has been conjectured that this approach does not capture the complexity of the phenomenon. A recent original experiment (Ibáñez-Berganza et al., Scientific Reports 9, $8364,2019)$ allowed different human subjects to navigate the face-space and "sculpt" their preferred modification of a reference facial portrait. Here we present an unsupervised inference study of the set of sculpted facial vectors in this experiment. We first infer minimal, interpretable and accurate probabilistic models (through Maximum Entropy and artificial neural networks) of the preferred facial variations, that encode the inter-subject variance. The application of such generative models to the supervised classification of the gender of the subject that sculpted the face reveals that it may be predicted with astonishingly high accuracy. We observe that the classification accuracy improves by increasing the order of the non-linear effective interaction. This suggests that the cognitive mechanisms related to facial discrimination in the brain do not involve the positions of single facial landmarks only, but mainly the mutual influence of couples, and even triplets and quadruplets of landmarks. Furthermore, the high prediction accuracy of the subjects' gender suggests that much relevant information regarding the subjects may influence (and be elicited from) their facial preference criteria, in agreement with the multiple motive theory of attractiveness proposed in previous works. 


\title{
Unsupervised inference approach to facial attractiveness
}

\author{
Miguel Ibáñez-Berganza ${ }^{1}$, Ambra Amico ${ }^{2}$, Gian Luca Lancia ${ }^{1}$, Federico \\ Maggiore $^{1}$, Bernardo Monechi ${ }^{3}$, and Vittorio Loreto ${ }^{1,3,4}$
${ }^{1}$ Sapienza University of Rome, Physics Department, Piazzale Aldo Moro 2, 00185 Rome, Italy.
${ }^{2}$ ETH Zurich, Chair of Systems Design, WEV G 212 Weinbergstrasse 56/58, 8092 Zurich.
${ }^{3}$ Sony Computer Science Laboratories, Paris, 6, rue Amyot, 75005, Paris, France.
${ }^{4}$ Complexity Science Hub, Josefstädter Strasse 39, A 1080 Vienna, Austria.

\author{
Corresponding author: \\ Miguel Ibáñez-Berganza ${ }^{1}$ \\ Email address: miguel.ibanezberganza@gmail.com
}

\begin{abstract}
The perception of facial attractiveness is a complex phenomenon which depends on how the observer perceives not only individual facial features, but also their mutual influence and interplay. In the machine learning community, this problem is typically tackled as a problem of regression of the subject-averaged rating assigned to natural faces. However, it has been conjectured that this approach does not capture the complexity of the phenomenon. A recent original experiment (Ibáñez-Berganza et al., Scientific Reports 9, 8364, 2019) allowed different human subjects to navigate the face-space and "sculpt" their preferred modification of a reference facial portrait. Here we present an unsupervised inference study of the set of sculpted facial vectors in this experiment. We first infer minimal, interpretable and accurate probabilistic models (through Maximum Entropy and artificial neural networks) of the preferred facial variations, that encode the inter-subject variance. The application of such generative models to the supervised classification of the gender of the subject that sculpted the face reveals that it may be predicted with astonishingly high accuracy. We observe that the classification accuracy improves by increasing the order of the non-linear effective interaction. This suggests that the cognitive mechanisms related to facial discrimination in the brain do not involve the positions of single facial landmarks only, but mainly the mutual influence of couples, and even triplets and quadruplets of landmarks. Furthermore, the high prediction accuracy of the subjects' gender suggests that much relevant information regarding the subjects may influence (and be elicited from) their facial preference criteria, in agreement with the multiple motive theory of attractiveness proposed in previous works.
\end{abstract}

\section{INTRODUCTION}

Human facial perception (of identity, emotions, personality dimensions, attractiveness) has been the subject of intense and multidisciplinary research in the last decades (Walker and Vetter (2016); Little et al. (2011); Leopold and Rhodes (2010)). In particular, facial attractiveness is a research topic that involves many different disciplines, from evolutionary biology and psychology to neuroscience (Bzdok et al. (2011); Hahn and Perrett (2014); Laurentini and Bottino (2014); Little (2014); Thornhill and Gangestad (1999)). Furthermore, it is an interesting case of study in the machine learning research community, as a paradigm of a complex cognitive phenomenon, ruled by complex and difficult to infer criteria. Indeed, the rules according to which a facial image will probably result pleasant are poorly known (Little et al. (2011)). The most relevant face-space variables in terms of which such rules should be inferred remain elusive as well (Laurentini and Bottino (2014)).

Many works have discussed, in the context of evolutionary biology, the validity of the so called natural selection hypothesis (Little et al. (2011); Rhodes (2006)), according to which the traits that we recognise as attractive are markers of a good phenotypic condition. Along with natural selection, also sexual selection and the handicap principle are known to play a role in facial attractiveness (Thornhill 
and Gangestad (1999)).

The evolutionary approach explains several aspects of the phenomenon, such as the impact in facial attractiveness of facial traits that are known to covary with a good phenotypic condition (averageness, symmetry, secondary sexual traits). Despite the success of the evolutionary approach, it is known that there are aspects of facial attractiveness which elude an evolutionary explanation. The natural selection hypothesis implies that the perception of attractiveness is mainly universal, species-typical. While a certain degree of universality has been assessed in many references, cultural and inter-person differences definitely play a role, beyond the species-typical criterion (Little (2014)). Several factors are known to influence the single subject idiosyncrasies, such as the subject's self- and other-rated attractiveness, genetic propensity, sexual orientation, and the menstrual cycle (see references in Oh et al. (2019b)).

Recently, many works have argued (the multiple motive hypothesis) that the evaluation of facial attractiveness is a complex process, influenced by the prior inference of semantic personality traits (such as dominance, extroversion or trustworthiness) that we consensually attribute to specific shape and luminance patterns in others' face (Oh et al. (2019a,b); Abir et al. (2017); Walker and Vetter (2016); Adolphs et al. (2016); Galantucci et al. (2014); Little (2014); Todorov and Oosterhof (2011); Oosterhof and Todorov (2008); Edler (2001); Cunningham et al. (1995)). According to this scenario, facial attractiveness is influenced by the single-subject relative inclination towards some fundamental personality traits. In the word of Oh et al. (2019a), individuals who highly value a personality trait, such as dominance, are likely to perceive faces that appear to possess the trait as attractive. This implies, in particular, that (A) the single subject preferred faces are expected to be, to some extent, distinguishable if characterised or inferred with sufficient accuracy, and (B) they are expected to reflect meaningful information regarding the subject.

The assessment of the validity of these hypotheses is, arguably, strongly influenced by the experimental precision with which the individuals' preferred faces can be characterised. While the natural selection hypothesis explains general aspects of facial attractiveness, if the experiments allow to resolve the single subjects' idiosyncrasies, more complex aspects and a strong subjectivity emerge (Hönekopp (2006); Oh et al. (2019b)). In particular, the subjectivity of facial attractiveness has been proven to be underestimated by the common experimental method from which most of the works draw their conclusions: the subjectaveraged rating assigned to several natural facial images (Hönekopp (2006)). Moreover, it has been argued that the average rating may suffer, as an experimental technique, the curse of dimensionality (the face-space being highly dimensional) and may consequently hinder the complexity and subjectivity of the phenomenon (Laurentini and Bottino (2014); Valentine et al. (2016); Ibáñez-Berganza et al. (2019)). Roughly half of the variance in attractiveness ratings has been attributed to idiosyncratic preferences, the other half to shared preferences (Hönekopp (2006)). It is a natural question whether such idiosyncratic proportion would result more prominent using an experimental method that bypasses the use of ratings.

This motivates the search for alternative experimental approaches. Ibáñez-Berganza et al. (2019) investigated the question (A) by means of an innovative experimental technique which permits the sampling of a single subject's preferred region in the face-space. ${ }^{1}$ As a matter of fact, the method allows the sampling of the subjects' preferred facial modifications with high precision. It is observed that, within the experimental precision (limited by the time that the subjects dedicate to the experiment), different subjects "sculpt" distinguishable facial modifications. Indeed, when repeating the experiment, they tend to sculpt facial modifications which are more similar to the ones that they already sculpted than to those sculpted by others, in $\sim 82 \%$ of the cases.

In the present work we present an inference analysis of the data collected in reference (IbáñezBerganza et al. (2019)), developing data-driven probabilistic generative models that describe the intersubject fluctuations around the average landmark positions. As previously stated, such fluctuations are expected to reflect and encode meaningful differences among experimental subjects. Indeed, we thereafter apply such models to the investigation of the aforementioned prediction (B); that is, whether we could elicit meaningful information regarding the subject from her/his preferred facial modifications. In particular, we address whether one may correctly predict the gender of the sculpting subject from such data. Many references have reported quantitative differences between male and female perception of

\footnotetext{
${ }^{1}$ The alternative experimental technique allows a given subject to seek her/his preferred variation of a reference facial portrait. Such variations differ only in a low-dimensional face-space of essential facial features. It is arguably the introduction of these two ingredients: the reduction of facial degrees of freedom and the possibility to efficiently explore the face-space (rather than rating facial images differing in many facial dimensions) that allows for a significant experimental distinction of different subject's criteria (see the SI for further details).
} 


\footnotetext{
${ }^{2}$ This inference scheme differs from the common one found in the facial attractiveness literature, specially in the machine learning papers. In these papers the main goal is the automatic rating of facial images, considered as a supervised inference problem (Laurentini and Bottino (2014)). The facial image is parametrised in a face-space vector $\mathbf{f}$, the inference goal consists in the regression $R(\mathbf{f})$ that reproduces at best the subject-averaged ratings $\left\langle R_{s}\right\rangle_{s}$ of a database $\left\{\mathbf{f}_{s}, R_{s}\right\}$. In the case of deep, hierarchical networks, which automatically perform feature selection, the raw image is used as an input to the learning algorithm instead of a face-space parametrisation $\mathbf{f}$. The resulting relevant features are, however, not immediately accessible.
}

facial attractiveness. Generally, males prefer smaller lower face area, higher cheekbones, larger mouths and eyes (see references in Little et al. (2011); Rhodes (2006); Thornhill and Gangestad (1999)). These facts are compatible with the results of Ibáñez-Berganza et al. (2019). In the present work we go one step further and demonstrate that gender, besides having an impact on the subject-averaged facial sculptures, can actually be predicted with almost certainty for single subjects, based on their facial modifications.

Our inference protocol allows the assessment of the relative influence of linear and non-linear correlations among facial coordinates in the classification, hence overcoming the black-box issue. In particular, we infer, in an unsupervised way, a collection of probabilistic generative models from the database of sculpted facial modifications $\mathscr{S}=\left\{\mathbf{f}^{(s)}\right\}_{s=1}^{S}$ (where $s$ is the subject index). Afterwards we assess the predictive power of the models, in two ways. (1) We evaluate the consistency of the simplest of such models, in order to ensure that all of them provide a faithful and economic description of the data. (2) The models are applied, when inferred from male/female data separately, to the supervised classification of the subject gender of a test-set and the results are compared with a powerful, specific algorithm for supervised classification, the random forest algorithm.

We infer, in particular, probabilistic models, $\mathscr{L}(\mathbf{f} \mid \boldsymbol{\theta})$, representing the probability density of a facial image with face-space vector $\mathbf{f}$ to be sculpted by any subject (given the reference facial portrait and the sculpture protocol). We have considered three generative models of unsupervised learning: two Maximum Entropy (MaxEnt) models, with linear and non-linear interactions among the facial coordinates, and the Gaussian Restricted Boltzmann Machine (GRBM) model of Artificial Neural Network (ANN). ${ }^{2}$

The models presented here are interpretable: the model parameters $\boldsymbol{\theta}$ provide information regarding the relative importance of the various facial distances and their mutual influence in the cognitive process of face perception, which are fundamental questions in the specific literature (Laurentini and Bottino (2014)). In particular, a comparison of the various models' efficiency highlights the relevance of the nonlinear influence (hence beyond proportions) of facial distances. Finally, this work provides a novel case of study, in the field of cognitive science, for techniques and methods in unsupervised inference and, in particular, a further application of the MaxEnt method (Jaynes (1957); Berg (2017); Nguyen et al. (2017)), otherwise extensively used in physics, systems neuroscience and systems biology (Lezon et al. (2006); Schneidman et al. (2006); Shlens et al. (2006); Bialek and Ranganathan (2007); Tang et al. (2008); Weigt et al. (2009); Roudi et al. (2009); Tkacik et al. (2009); Stephens and Bialek (2010); Mora et al. (2010); Morcos et al. (2011); Bialek et al. (2012); Martino and Martino (2018)).

The structure of the article is as follows. The inference models will be presented in sec. 2 , along with some key methodological details. In sec. 3 we analyse the results following our objectives $(1,2)$ described above: we first evaluate the quality of our models as generative models of the set of facial modifications in (Ibáñez-Berganza et al. (2019)). Afterwards, we perform a further assessment in which we apply the generative models to the classification of the gender of the subjects from their sculpted facial vectors, and compare the results with that of a purely supervised learning algorithm. We draw our conclusions in sec. 4.

\section{MATERIALS AND METHODS}

\subsection{Description of the database}

We analyse the dataset $\mathscr{S}$ described by Ibáñez-Berganza et al. (2019). In such experiments, each subject was allowed to sculpt her/his favorite deformation of a reference portrait (through the interaction with an software which combines image deformation techniques with a genetic algorithm for the efficient search in the face-space). The set of selected images are, hence, artificial, though completely realistic, variations of a common reference portrait (corresponding to a real person). In such a way, only the geometric positions of the landmarks are allowed to vary, the texture degrees of freedom are fixed (and correspond to the reference portrait RP1 (taken from the Chicago database, see (Ma et al. (2015)) and fig. 1). This representation of the face is, hence, rooted on a decoupling of geometric (also called shape) and texture 
(also called reflectance) degrees of freedom (Laurentini and Bottino (2014)). ${ }^{3}$ See Description of the E1 experiment in the SI for a more detailed descriptions of the experimental protocol in (Ibáñez-Berganza et al. (2019)).

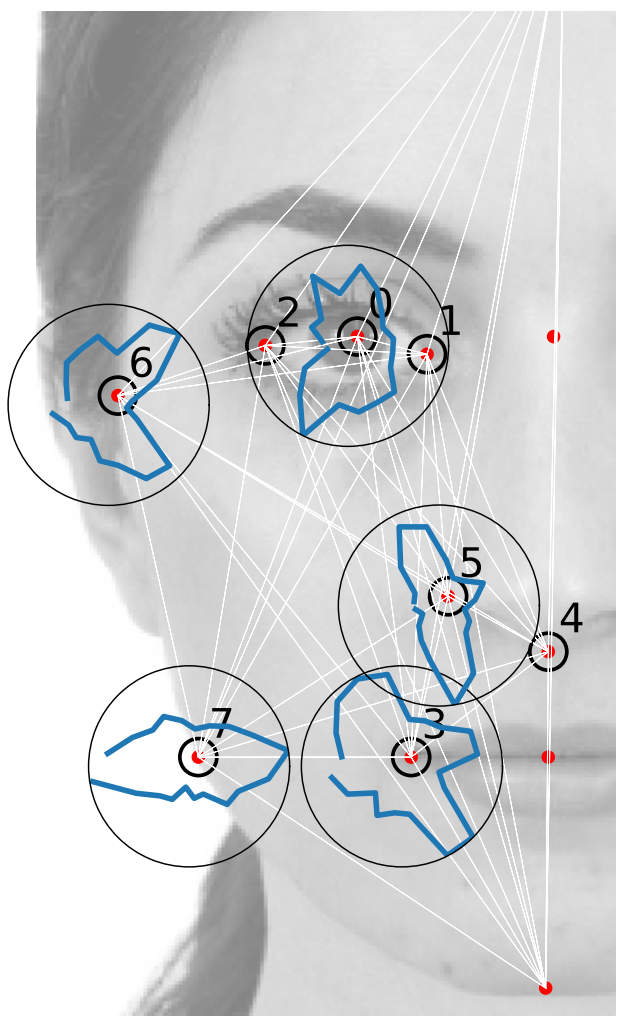

Figure 1. Facial landmarks $i=0, \ldots, 7$ whose $2 \mathrm{D}$ coordinates $\vec{r}_{i}$ constitute the face space (signaled with black circles). Their position in the figure correspond to the average position, $\left\langle\vec{r}_{i}\right\rangle$. The background image corresponds to the texture degrees of freedom of the reference portrait. The blue lines are polar histograms $h(\phi)$ (the radius is proportional to $h(\phi)$ ) corresponding to the experimental distribution of angle landmark fluctuations around their average position.

The database consists in the set of landmark geometric coordinates $\mathscr{S}=\left\{\mathbf{r}^{(s)}\right\}_{s=1}^{S}$, where $s$ is the facial vector index corresponding to the $\mathscr{N}=28$ vectors sculpted by each of the $n_{\mathrm{s}}=95$ experimental subjects (hence: $\left.S=n_{\mathrm{s}} \mathscr{N}=2660\right) .{ }^{4}$ We will call $\mathbf{r}^{(s)}=\left(r_{(\mathrm{x}, 1)}^{(s)}, \ldots, r_{(\mathrm{x}, n)}^{(s)}, r_{(\mathrm{y}, 1)}^{(s)}, \ldots, r_{(\mathrm{y}, n)}^{(s)}\right)$ the vector whose $2 n$ components are the $(\mathrm{x}, \mathrm{y})$ Cartesian coordinates of a set of $n=8$ landmarks, in units of the facial height. Such landmarks (those signaled with an empty circle in figure 1) are a subset of the set of landmarks used for the image deformation in (Ibáñez-Berganza et al. (2019)) (signaled with red points in figure 1). We will also refer to the 2D Cartesian vector of the $i$-th landmark as $\vec{r}_{i}=\left(\mathrm{x}_{i}, \mathrm{y}_{i}\right)$, and define the fluctuations of the landmark positions with respect to their average value as $\vec{\Delta}_{i}=\vec{r}_{i}-\left\langle\vec{r}_{i}\right\rangle$, where $\langle\cdot\rangle$ denotes the experimental average, $\langle\cdot\rangle=(1 / S) \sum_{s} \cdot$. Analogously, $\Delta^{(s)}=\mathbf{r}^{(s)}-\langle\mathbf{r}\rangle$. An important aspect of the dataset is that even the coordinates of the restricted set of $n=8$ landmarks, $\mathbf{r}^{(s)}$, are redundant and

\footnotetext{
${ }^{3}$ Many works exploit the geometric/texture decoupling in artificial facial images to study separately the effect of both kinds of coordinates. It is also a natural strategy of dimensionality reduction of the human face, that has been observed to be implemented in both the neural code for facial identification in the brain and by artificial neural networks (Chang and Tsao (2017); Higgins et al. (2020)). In (Ibáñez-Berganza et al. (2019)), we combine this separation with the use of completely realistic images, thus eliminating the bias that artificial images are known to induce in experiments (Balas and Pacella (2015); Oh et al. (2019b)).

${ }^{4}$ Actually, the database $\mathscr{S}=\left\{\mathbf{r}^{(v, i)}\right\}$ is composed by $S=n_{\mathrm{s}} \times \mathscr{N}$ facial vectors labelled by a single index $s=1, \ldots, S$ or, alternatively, by a tuple of indices $(v, i)\left(v=1, \ldots, n_{\mathrm{s}}, i=1, \ldots, \mathscr{N}, n_{\mathrm{s}}=95, \mathscr{N}=28\right)$ referring to the $i$-th facial vector sculpted by the $v$-th subject (in a single genetic experiment, see (Ibáñez-Berganza et al. (2019))). In the SI we present a detailed analysis of the error estimation over the dataset, distinguishing inter- and intra-subject fluctuations. The last ones are, in principle, an artifact of the sculpting process, but they may encode part of the subject's idiosyncrasy. Similarly, the inferred models may be conceived to account for intra- and inter-subject, or only for inter-subject correlations (see the SI).
} 
depend on 10 coordinates only, due to the presence of $2 n-10=6$ constraints that result from the very definition of the face-space. Such constraints are described in detail in the SI.

\subsection{Unsupervised inference}

We now present some non-technical notion of unsupervised learning. In the next subsection we will describe the probabilistic models with which we describe the dataset. These are generative models that induce a likelihood probability density $\mathscr{L}(\cdot \mid \boldsymbol{\theta})$ over the space of facial vectors $\Delta$. The meaning of $\mathscr{L}(\boldsymbol{\Delta} \mid \boldsymbol{\theta})$ is that the probability of finding a facial vector in an interval of facial vectors $\mathbf{I}$ is given by $\int_{\mathbf{I}} \mathscr{L}(\boldsymbol{\Delta} \mid \boldsymbol{\theta}) \mathrm{d} \boldsymbol{\Delta}$. The probabilistic model $\mathscr{L}$ represents a generalisation of the database, from which it is not unambiguously elicited. Indeed, a probabilistic model of the data depends both on the functional form of $\mathscr{L}$ (often called simply the model), and on the learning algorithm, or the protocol with which its parameters $\boldsymbol{\theta}$ are inferred form the data.

For the learning algorithm, in the present work we adopt the Maximum Likelihood principle. We fix the parameters to the value that maximises the database likelihood: $\boldsymbol{\theta}^{*}=\arg \max _{\boldsymbol{\theta}} \prod_{s} \mathscr{L}\left(\boldsymbol{\Delta}^{(s)} \mid \boldsymbol{\theta}\right)$, where the product is over all the samples in the database (please, see Inter-and intra-subject correlations and errors in the SI for further information at this regard).

The functional form is given by the kind of unsupervised model. In this article we will consider three models (2- and 3-MaxEnt, GRBM), that will be described in the following subsections. The 2- and 3-MaxEnt models follows from the Maximum Entropy principle (Jaynes (1957); Berg (2017); Nguyen et al. (2017); Martino and Martino (2018)), which provides the functional form of the probability distribution $\mathscr{L}(\cdot \mid \boldsymbol{\theta})$ that exhibits maximum entropy and, at the same time, is consistent with the average experimental value of some observables of the data, $\langle\Sigma\rangle$, that will be called sufficient statistics. $\mathscr{L}$ must satisfy $\langle\Sigma\rangle_{\mathscr{L}}=\langle\Sigma\rangle$, where $\langle\cdot\rangle_{\mathscr{L}}$ refers to the expected value according to $\mathscr{L}$. In other words, $\mathscr{L}$ is the most general probability distributions constrained to exhibit a fixed expectation value of $\langle\Sigma\rangle_{\mathscr{L}}$ (and this value, under the Maximum Likelihood prescription, is given by the corresponding experimental value). The precise choice of the sufficient statistics determines the functional form of $\mathscr{L}(\boldsymbol{\Delta} \mid \boldsymbol{\theta})$. A more detailed description is given in the next section and in section Introduction to the Maximum Entropy principle: Correlations vs effective interactions of the SI.

\subsection{The Maximum Entropy models}

We propose two MaxEnt probabilistic generative models of the set of selected faces, inferred from the dataset $\mathscr{S}$. In the case of the Gaussian or 2-MaxEnt model, the sufficient statistics is given by the $2 n$ averages $\left\langle\Delta_{\mu}\right\rangle$ and by the $2 n \times 2 n$ matrix of horizontal, vertical and oblique correlations among couples of vertical and horizontal landmark coordinates, whose components are $C_{\mu \nu}=\left\langle\Delta_{\mu} \Delta_{v}\right\rangle$. In these equations, the $2 n$ Greek indices $\mu=i, c_{i}$ denote the $c_{i}=\mathrm{x}, \mathrm{y}$ coordinates of the $i$-th landmark. The 2-MaxEnt model probability distribution takes the form (see the SI) of a Maxwell-Boltzmann distribution, $\mathscr{L}(\boldsymbol{\Delta} \mid \boldsymbol{\theta})=\frac{1}{Z} \exp (-H(\boldsymbol{\Delta} \mid \boldsymbol{\theta}))$. In this equation, $Z$ is a normalising constant (the partition function, in the language of statistical physics) depending on $\boldsymbol{\theta}$, and $H=H_{2}$ (the Hamiltonian) is the function:

$$
H_{2}\left(\boldsymbol{\Delta} \mid \boldsymbol{\theta}_{2}\right)=\frac{1}{2} \boldsymbol{\Delta}^{\dagger} \cdot J \cdot \boldsymbol{\Delta}+\mathbf{h}^{\dagger} \cdot \boldsymbol{\Delta}
$$

The model depends on the parameters $\boldsymbol{\theta}_{2}=\{J, \mathbf{h}\}$, or the $2 n \times 2 n$ matrix of effective interactions $J$ and the $2 n$ vector of effective fields, $\mathbf{h}$. Due to the symmetry of matrix $J$, the number of independent parameters in the 2-MaxEnt model is $D+D(D+1) / 2$, where $D=2 n$ is the dimension of the vectors of landmark coordinates $\boldsymbol{\Delta}$. The value of these parameters is such that the equations $\langle\boldsymbol{\Delta}\rangle=\langle\boldsymbol{\Delta}\rangle_{\mathscr{L}}$ and $\left\langle\Delta_{\mu} \Delta_{v}\right\rangle_{\mathscr{L}}=C_{\mu \nu}$ are satisfied. This is equivalent to require that $\boldsymbol{\theta}_{2}$ are those that maximise the likelihood of the joint $\mathscr{L}$ over the database $\mathscr{S}$ (the Maximum Likelihood condition). The solution of such an inverse problem is (see SI): $J=C^{-1}, \mathbf{h}=J \cdot\langle\boldsymbol{\Delta}\rangle$, and $Z=(2 \pi)^{n} \exp \left(\mathbf{h}^{\dagger} \cdot J^{-1} \cdot \mathbf{h} / 2\right)(\operatorname{det} J)^{-1 / 2}$, where the -1 power in equation $J=C^{-1}$ denotes the pseudo-inverse operation, or the inverse matrix disregarding the null eigenvalues induced by the database constraints (see SI). The 2-MaxEnt model is equivalent in the present case, in which the coordinates $\Delta_{\mu}$ are real numbers, to a Principal Component Analysis and $\mathscr{L}$ is in this case a multi-variate Normal distribution.

We will define as well the 3-MaxEnt model. In this case, the sufficient statistics is given by averages $\left\langle\Delta_{\mu}\right\rangle$, pairwise correlations $C_{\mu \nu}$, and correlations among 3-landmark coordinates, $C_{\mu \nu \kappa}^{(3)}=\left\langle\Delta_{\mu} \Delta_{v} \Delta_{\kappa}\right\rangle$. The 
3-MaxEnt model probability distribution function takes the form of a Maxwell-Boltzmann distribution multiplied by a regularisation term ensuring that it is normalisable:

$$
\mathscr{L}(\cdot \mid \mathbf{h}, J, Q)=\frac{1}{Z_{3}} e^{-\left[H_{2}\left(\cdot \mid \boldsymbol{\theta}_{2}\right)+H_{3}(\cdot \mid Q)\right]} \mathrm{H}(\cdot \mid B)
$$

where $\mathrm{H}(\cdot \mid B)$ is a multivariate Heaviside function, equal to one for vectors $\Delta$ lying in the hypercube $-B \leq$ $\Delta_{\mu} \leq B$ for all $\mu$ and zero otherwise; $Z_{3}$ is the normalising factor, and the Hamiltonian is $H=H_{2}+H_{3}$, where $H_{2}$ given by equation (1) and $H_{3}$ by:

$$
H_{3}(\boldsymbol{\Delta} \mid Q)=\frac{1}{6} \sum_{\mu \nu \kappa} \Delta_{\mu} \Delta_{v} \Delta_{\kappa} Q_{\mu \nu \kappa}
$$

Besides $\mathbf{h}$ and $J$, the non-linear MaxEnt model depends on a further tensor of three-wise interaction constants among triplets of landmark coordinates. Consequently, the number of independent parameters is $[D]+[D(D+1) / 2]+\left[\left(D^{3}-D^{2}\right) / 6+D\right]=D^{3} / 6+D^{2} / 3+5 D / 2$.

The solution of the inverse problem for the non-linear MaxEnt model does not take a closed analytic form. The maximum likelihood value of the parameters $(\mathbf{h}, J, Q)$ is numerically estimated by means of a deterministic gradient ascent algorithm (see Monechi et al. (2020)). A detailed explanation of the learning protocol may be found in the SI (see Learning in the non-linear MaxEnt model). Before inferring the data with the non-linear models (3-MaxEnt and GRBM) we have eliminated a subset of 6 redundant coordinates from the original $2 n$ coordinates. The data has been standardised in order to favor the likelihood maximisation process. The value of $B$ has been chosen to be $B=6$, so that the probability distribution function is nonzero only in an hypercube whose side is six times the standard deviation of each standardised variable.

\subsection{The Restricted Boltzmann Machine model for unsupervised inference.}

We have learned the data with the (Gaussian-Binary) Restricted Boltzmann Machine (GRBM) model of unsupervised inference (Wang et al. $(2012,2014)$ ). It is a 2-layer unsupervised ANN, a variant, processing input real vectors, of the binary-binary RBM model. The model induces a probability distribution $\mathscr{L}(\boldsymbol{\Delta} \mid \boldsymbol{\theta})=\sum_{\mathbf{h}} p(\boldsymbol{\Delta}, \mathbf{h} \mid \boldsymbol{\theta})$ which is obtained by the marginalisation, over a set of $N_{\mathrm{h}}$ binary hidden variables (or hidden neurons), $h_{j} \in\{0,1\}, j=1, \ldots, N_{\mathrm{h}}$, of a joint probability distribution $p$ :

$$
\mathscr{L}(\boldsymbol{\Delta} \mid \boldsymbol{\theta})=\sum_{\mathbf{h}} p(\boldsymbol{\Delta}, \mathbf{h} \mid \boldsymbol{\theta}), \quad p(\mathbf{v}, \mathbf{h} \mid \boldsymbol{\theta})=\frac{1}{Z_{\boldsymbol{\theta}}} e^{-E(\mathbf{v}, \mathbf{h} \mid \boldsymbol{\theta})}
$$

The interaction among visible and hidden variables, and the dependence of $p(\boldsymbol{\Delta}, \mathbf{h} \mid \boldsymbol{\theta})$ on all its arguments is described by an energy function $E$ that couples hidden to visible neurons. $E$ is defined in terms of a set of parameters $\boldsymbol{\theta}$ consisting, among others, on the $D \times N_{\mathrm{h}}$ matrix of synaptic weights among visible (input) and hidden variables. Although $E$ presents only a linear coupling among $\mathbf{v}$ and $\mathbf{h}$, the marginalisation over binary hidden neurons actually induce nonlinear effective couplings at all orders among the visible variables $\mathbf{v}$ (or $\boldsymbol{\Delta}$ ), couplings that may be accessed from the network parameters $\boldsymbol{\theta}$ (MacKay (2003); Cossu et al. (2019)).

We have employed the open-source software (Melchior (2017)) for the efficient learning of GRBM. The learning protocol and parameters are described in detail, along with an introduction to the GRBM model, in the SI, see: Learning the database with the Gaussian Restricted Boltzmann Machine.

\section{RESULTS}

We will now present an assessment of the description of the database according to the inference models described in the precedent section. In sec. 3.1 we will argue that the 2-MaxEnt model is a faithful representation of the dataset, and that only the nonlinear models predict the subject's gender when applied to such supervised inference task. Finally, in subsection 3.2, we will argue that the matrix of effective interactions $J$ provides interpretable information, beyond the raw information present in the raw experimental measure $C$. 

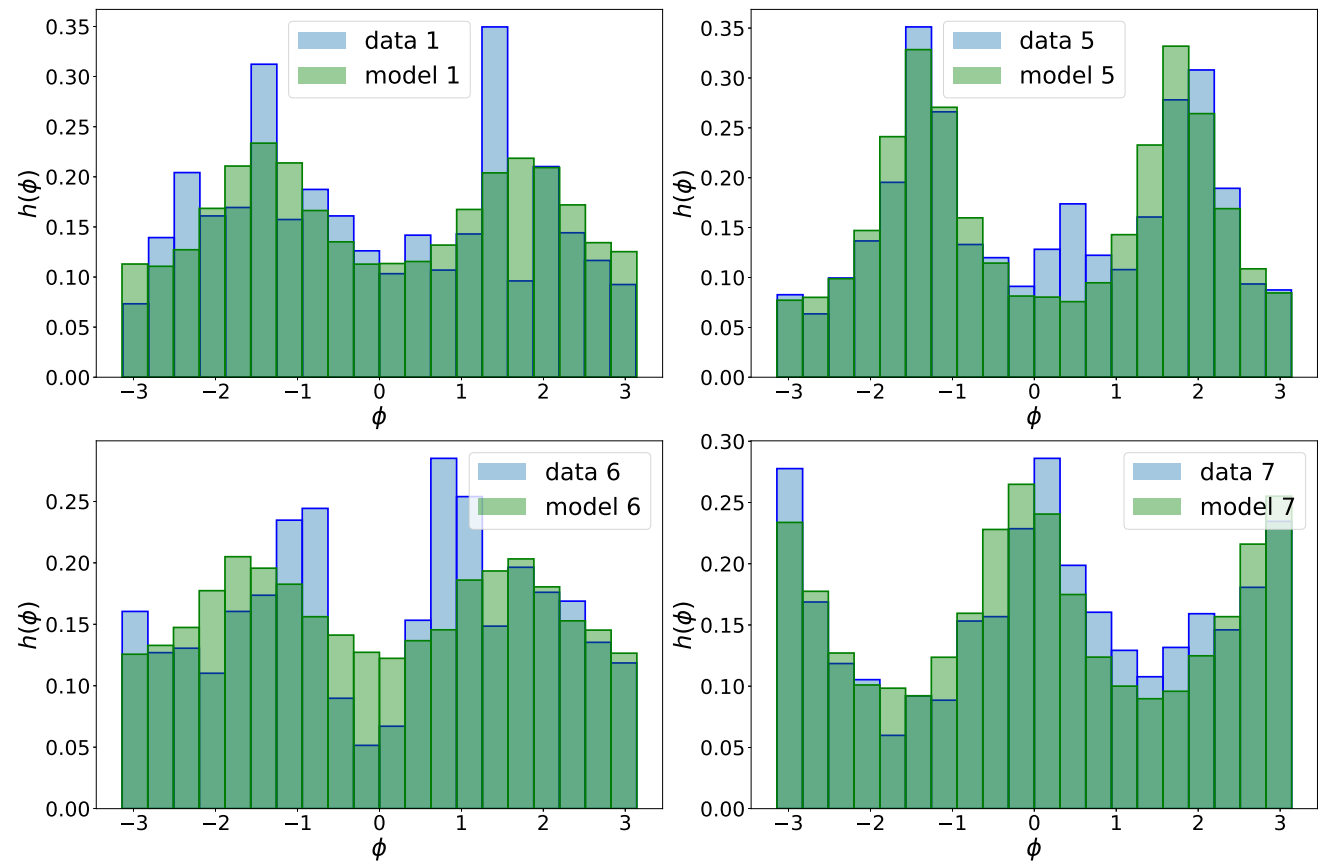

Figure 2. Comparison among empirical $\left(\mathrm{h}^{(i)}(\phi)\right)$ and theoretical $\left(\mathrm{h}_{\mathrm{t}}^{(i)}(\phi)\right)$ histograms of angle landmark fluctuations, for several landmarks, $i=1,5,6,7$, see fig. 1 (from left to right, from top to bottom). The empirical histograms $\mathrm{h}^{(i)}(\phi)$ represent the probability density of empirical displacements $\vec{\Delta}_{i}^{(s)}$ of the $i$-th landmark along an axes which subtends an angle $\phi \in(-\pi, \pi)$ with the horizontal axis. These histograms are presented for all the landmarks also in fig. 1, under the form of polar histograms. $h_{t}^{(i)}(\phi)$ is the theoretical prediction of the same quantity, obtained by sampling data from the inferred $\mathscr{L}(\cdot \mid \boldsymbol{\theta})$.

\subsection{Quality of the MaxEnt models as generative models 3.1.1 Histograms of single landmark-angle fluctuations}

The quality of the 2-MaxEnt generative model as a faithful description of the database may be evaluated by the extent to what the model $\mathscr{L}$ reproduces observables $O$ that it is not required to reproduce by construction. In other words, observables that cannot be written in terms of couples and triplets of coordinates $\Delta_{\alpha}$. The model is faithful in the extent to what $\langle O\rangle \simeq\langle O\rangle_{\mathscr{L}}$.

The $i$-th landmark coordinates $\vec{\Delta}_{i}$ tend to fluctuate in the database with respect their average position $\left\langle\vec{\Delta}_{i}\right\rangle=\overrightarrow{0}$. As a nonlinear observable $O$ we will consider the angle that the $i$-th landmark fluctuation $\vec{\Delta}_{i}$ forms with the x-axis. This quantity will be referred to as $\phi_{i}^{(s)}=\arctan \left(\Delta_{i, \mathrm{y}}^{(s)} / \Delta_{i, \mathrm{x}}^{(s)}\right)$. In figs. 1,2 , we report the empirical histogram of angles, $\mathrm{h}\left(\phi_{i}\right)$ for some landmarks $i$. Remarkably, some landmarks' angle distribution exhibit local maxima, probably reflecting their tendency to follow the direction of some inter-landmark segments (as it is apparent for the 3-rd and 6-th landmark's in figure 1). ${ }^{5}$ We have compared the empirical histograms with the theoretical $\phi_{i}$ distributions according to the model. These have been obtained as the angle histograms of a set of $S$ vectors $\Delta$ sampled from the inferred distribution $\mathscr{L}(\cdot \mid \boldsymbol{\theta})$ (see fig. 2). The 2-MaxEnt model satisfactorily reproduces most of the landmark angle distributions. The empirical angle distribution, in other words, is reasonably well reproduced by the theoretical distribution $\mathrm{h}_{\mathrm{t}}(\varphi)=\int \mathrm{d} \boldsymbol{\Delta} \mathscr{L}(\boldsymbol{\Delta} \mid \boldsymbol{\theta}) \delta(\phi(\boldsymbol{\Delta})-\varphi)$.

It is important to remark that the model-data agreement on $\mathrm{h}\left(\phi_{i}\right)$ is observed also for large values of $\left|\phi_{i}\right| \in(0, \pi)$ (see fig. 2), and not only for small values of $\left|\phi_{i}\right|$, for which it approximately becomes $\left|\Delta_{i, y} / \Delta_{i, x}\right|$ (whose average is related to the correlation $C_{\alpha \beta}$, see SI).

We conclude that, very remarkably, a highly non-linear observable as $\phi$ is well described by the 2-MaxEnt model, albeit it has been inferred from linear (pairwise) correlations only. In this sense, the

\footnotetext{
${ }^{5}$ Interestingly, such local maxima seem to be oriented along inter-landmark segments eventually involving landmarks which are not described in the facial vectors $\vec{\Delta}$ : the landmarks $\ell_{0}$ and $\ell_{18}$, see the SI.
} 


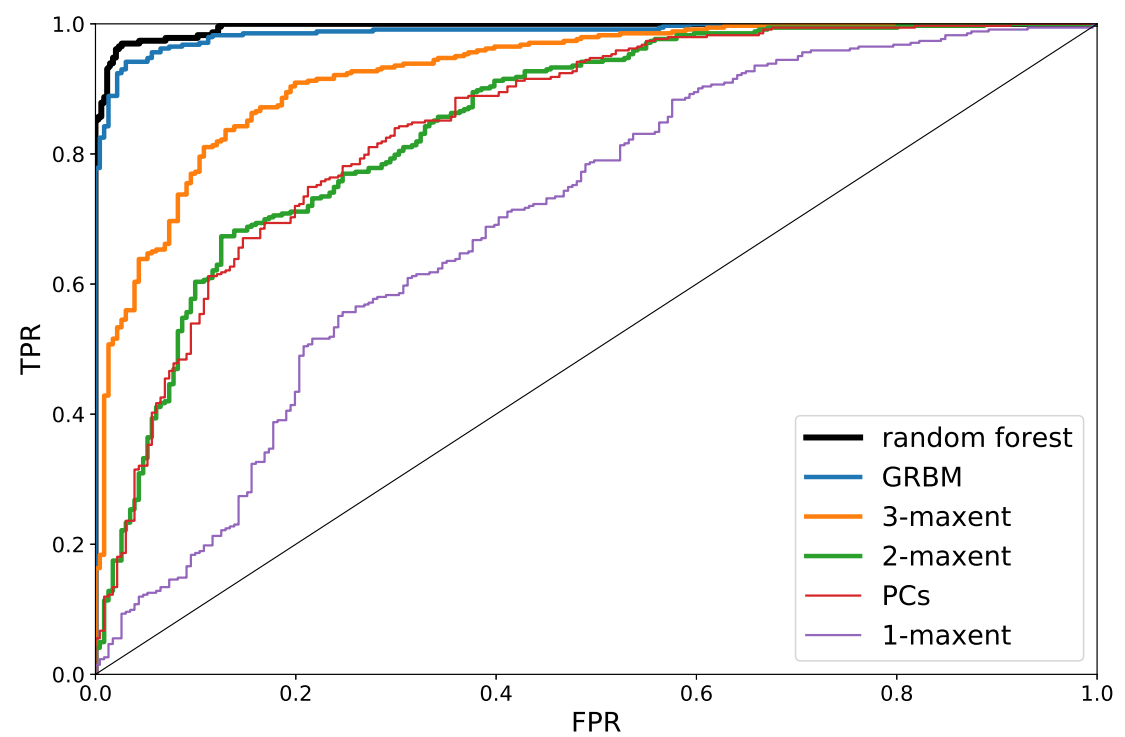

Figure 3. True Positive Rate (TPR) versus False Positive Rate (FPR) corresponding to the Receiver Operating Characteristic (ROC) curves associated to the gender classification. Different ROC curves correspond to different algorithms. PC's refers to a t-Student test of the difference in the principal components of a vector with respect to their average value in the $A, B$ sets.

2-MaxEnt model is a faithful and economic description of the dataset. This picture is confirmed by the results of the following section which suggest, however, that a description of the gender differences in the dataset require taking into account effective interactions of order $p>2$.

\subsubsection{Performance of the MaxEnt model in a classification task}

We now further evaluate the quality of the 2- and 3-MaxEnt models by assessing their efficiency to classify a test database of vectors in two disjoint subsets $\mathscr{S}=\mathscr{S}_{A} \cup \mathscr{S}_{B}$ corresponding to the gender of the subject that sculpted the facial vector in (Ibáñez-Berganza et al. (2019)). We compare such efficiency with that of the GRBM model of ANN (see Wang et al. (2012, 2014), sec. 2 and the SI). This comparison allows to assess the relative relevance of products of $p$-facial coordinates $\Delta_{\alpha}$ in the classification task: averages $(p=1)$, pairwise correlations $(p=2)$, and non-linear correlations of higher, $p>2$ order (modelled by the 3-MaxEnt and GRBM models only).

The dataset is divided in two disjoint classes $\mathscr{S}_{A}, \mathscr{S}_{B}$. Afterwards, both $\mathscr{S}_{A, B}$ are divided in trainingand test- sets (20\% and $80 \%$ of the elements of $\mathscr{S}_{A, B}$, respectively), and inferred the $A$ and $B$ training sets separately, with the MaxEnt and GRBM models. This results in six $(\{2,3, G\} \times\{A, B\})$ sets of parameters $\boldsymbol{\theta}_{A, B}^{2,3, G}$, where the super-index refers to the model. Given a vector $\Delta$ belonging to the $A$ or $B$ test set, the score $\mathbf{s}(\boldsymbol{\Delta})=\ln \mathscr{L}\left(\boldsymbol{\Delta} \mid \boldsymbol{\theta}_{A}\right)-\ln \mathscr{L}\left(\boldsymbol{\Delta} \mid \boldsymbol{\theta}_{B}\right)$ is taken as the estimation of the model prediction for $\Delta \in \mathscr{S}_{A}$. The resulting Receiver Operating Characteristic (ROC) curves (Murphy (2012)) are shown in fig. 3 for the various models considered. ${ }^{6}$

Considering only the averages $\langle\boldsymbol{\Delta}\rangle$ as sufficient statistics (or, equivalently, inferring only the fields $\mathbf{h}$ and setting $J_{i j}=\sigma_{i}^{-2} \delta_{i j}$ in equation 1) results in a poor, near-casual classification (specially in the most interesting region of the ROC curve, for small FPR and large TPR), see figure 3. The 2-MaxEnt model allows, indeed, for a more efficient classification. Rather remarkably, with the 3-MaxEnt and GRBM models the classification accuracy gradually increases. We interpret this as an indication of the fact that

\footnotetext{
${ }^{6}$ These consist in a scatter plot with the fraction of true positive classifications (TPR) in the $\mathscr{S}_{A}$ test-set versus the fraction of false positive classifications (FPR) in the $\mathscr{S}_{B}$ test-set, where each point corresponds to a different soil $\delta$ over the estimator $\mathrm{s}(\boldsymbol{\Delta}) \lessgtr \delta$ that we use to assign whether the model predicts that $\Delta$ belongs to $A$ or $B$. The curve is invariant under reparametrizations of $\mathrm{s} \rightarrow f(\mathrm{~s})$ defined by any monotone function $f$.
} 


\footnotetext{
${ }^{7}$ As we explain in the SI, the non-Gaussian correlations of order 3 present in the dataset are, at least partially, not of cognitive origin, but due to an artifact of the numerical algorithm allowing subjects to sculpt their preferred facial vectors. However, we believe that the non-linear effective interactions that we infer do reflect the existence of non-linear operations playing a role in believe that the non-linear effective interactions that we infer do reflect the existence of non-linear operations playing a role in
the cognitive process of facial evaluation. This is suggested by the fact that the introduction of non-linear effective interactions drastically improves the gender classification.
}

non-linear effective interactions at least of fourth order are necessary for a complete description of the database. For completeness, we have included a comparison with the Random Forest (RF) algorithm (Murphy (2012)). As shown in fig. 3, RF achieves the highest classification accuracy (auROC $=0.995$, see $\mathrm{SI})$. We notice that this does not imply that the unsupervised models are less accurate: the RF algorithm is advantaged, being a specific model trained to classify at best the $A, B$ partitions, not to provide a generative model of the $A$ and $B$ partitions separately.

We report the maximal accuracy scores for all the algorithms: RF (0.971); GRBM (0.952); 3MaxEnt (0.865); 2-MaxEnt (0.764); 1-MaxEnt (0.680). The 2-MaxEnt model efficiency is, as expected, compatible with that of a t-Student test regarding the differences in the principal component values of $A$ and $B$ vectors, see fig. 3. See the auROC scores of all the algorithms in the SI.

We conclude that, on the one hand, the subjects' gender strikingly determines her/his preferred set of faces, to such an extent that it may be predicted from the sculpted facial modification with an impressively high accuracy score (Murphy (2012)): a 97.1\% of correct classifications. On the other hand, the relative efficiency of various models highlights the necessity of non-linear interactions for a description of the differences among male and female facial preference criterion in this database. Arguably, such nonlinear functions play also a role in the cognitive process of facial perception. The criterion with which the subjects evaluate and discriminate facial images seems to involve not only proportions $r_{\alpha} / r_{\beta}$ (related to the pairwise correlations $C_{\alpha \beta}$, see SI), but also triplets and quadruplets of facial coordinates influencing each other (yet, see the SI for an alternative explanation) ${ }^{7}$.

It is believed that the integration of different kinds of facial variables (geometric, feature-based versus texture, holistic, see (Trigueros et al. (2018); Valentine et al. (2016))) improve the attractiveness inference results, suggesting that both kinds mutually influence each other in attractiveness (Eisenthal et al. (2006); Xu et al. (2017); Laurentini and Bottino (2014); Ibáñez-Berganza et al. (2019)). The present results indicate that, even restricting to geometric coordinates (at fixed texture degrees of freedom), it is necessary a holistic approach, in the sense that it considers the mutual influence of many geometric coordinates.

Actually, our results indicate that pairwise influence of geometric coordinates are enough for a fair and economic description of the database (the 2-MaxEnt model predicts nonlinear observables, beyond the empirical information with which it has been fed). However, the differences among the facial vectors sculpted by males and females are not only encoded in pairwise correlations among geometric coordinates. Facial vectors reveal the gender of the sculpting subject with almost certainty only when non-linear models are used.

\subsection{Analysis of the matrix of effective interactions}

We now show that the generative models may provide directly interpretable information. This is an advantage of the MaxEnt method, whose parameters, the effective interaction constants, may exhibit an interpretable significance. We prove, in particular, that at least the matrix of effective interactions $J$ admits an interpretation in terms of "resistance" (elastic constant) of inter-landmark segment distances and angles to differ with respect to their average or preferred value.

The 2-MaxEnt model admits an immediate interpretation. The associated probability density $\mathscr{L}(\boldsymbol{\Delta} \mid \boldsymbol{\theta})=$ $\exp \left(-H_{2}(\boldsymbol{\Delta} \mid \boldsymbol{\theta})\right) / Z$ formally coincides with a Maxwell-Boltzmann probability distribution of a set of $n$ interacting particles in the plane (with positions $\vec{\Delta}_{i}, i=1, \ldots, n$ ), subject to the influence of a thermal bath at constant temperature. Each couple $i, j$ of such fictitious set of particles interacts through an harmonic coupling that corresponds to a set of three effective, virtual springs with non-isotropic elastic constants, $J^{(\mathrm{xx})}{ }_{i j}, J^{(\mathrm{yy})}{ }_{i j}, J^{(\mathrm{xy})}{ }_{i j}$ corresponding (see equation 1) to horizontal, vertical and oblique displacements, $\Delta_{i, \mathrm{x}}-\Delta_{j, \mathrm{x}}, \Delta_{i, \mathrm{y}}-\Delta_{j, \mathrm{y}}$, and $\Delta_{i, \mathrm{x}}-\Delta_{j, \mathrm{y}}$, respectively.

The inferred effective interactions are more easily interpretable if one considers, rather than their $x \mathrm{x}$, yy and xy components, the longitudinal and torsion effective interactions, $J_{i j}^{\|}$and $J_{i j}^{\perp}$, respectively. The longitudinal coupling $\left|J_{i j}^{\|}\right|$may be understood (see the SI for a precise definition) as the elastic constant corresponding to the virtual spring that anchors the inter- $i j$ landmark distance to its average value, $\left\langle r_{i j}\right\rangle$ (where $\vec{r}_{i j}=\vec{r}_{j}-\vec{r}_{i}$ ). In its turn, the torsion interaction $\left|J_{i j}^{\perp}\right|$ is the elastic constant related to fluctuations of 
$\vec{r}_{i j}$ along the direction normal to $\vec{r}_{i j}$ or, equivalently, to fluctuations of the $i j$-segment angle, with respect to its average value that we will call $\alpha_{i j}=\arctan \left(\left\langle r_{i j, y}\right\rangle /\left\langle r_{i j, \mathrm{x}}\right\rangle\right)$.

In fig. 4-A,B we show the quantities $\left|J_{i j}^{\|}\right|$and $\left|J_{i j}^{\perp}\right|$ for those couples $i, j$ presenting a statistically significant value (for which the $\mathrm{t}$-value $t_{i j}=\left|J_{i j}\right| / \sigma_{J_{i j}}>1$ (a description of the calculation protocol of the bootstrap error $\sigma_{i j}$ may be found in the SI). The width of the colored arrow over the $i, j$ segment is proportional to $\left|J_{i j}^{\|}\right|$(blue arrows in fig. 4-A) and $\left|J_{i j}^{\perp}\right|$ (red arrows in fig. 4-B). We notice that there exist inter-landmark segments for which $\left|J_{i j}^{\|}\right|$is significant while $\left|J_{i j}^{\perp}\right|$ is not (as the 0,4 or the 5,6 segments) and vice-versa (as the 6,7 and 2,5). This suggests that $\left|J_{i j}^{\|}\right|,\left|J_{i j}^{\perp}\right|$ actually capture the cognitive relative relevance of distance fluctuations around $\left\langle r_{i j}\right\rangle$, and of angle fluctuations around $\alpha_{i j}$.

We remark that the prominent importance of the inter-segment angles $i j$ highlighted in fig. 4-B is fully compatible with the analysis presented in (Ibáñez-Berganza et al. (2019)) at the level of the oblique correlation matrix $C^{(\mathrm{xy})}$, and it goes beyond, as far as it quantitatively assess their relative relevance. As we will see in the next subsection, such information cannot be retrieved from the experimental matrix $C$ only.

In the SI we explain in more detail the analogy with the system of particles. We also analyse the dependence of the torsion and longitudinal effective interactions, $\left|J_{i j}^{\|}\right|$and $\left|J_{i j}^{\perp}\right|$, with the average distance and angle of the $i j$ inter-landmark segment, showing that there is a moderate decreasing trend of $\left|J_{i j}^{\|}\right|$with $\left\langle r_{i j}\right\rangle$. This fact admits an interpretation: large inter-landmark distances are less "locked" to their preferred value with respect to shorter distances. Very interestingly, such trend is less evident for $\left|J_{i j}^{\perp}\right|:$ the relative relevance of the inter-landmark angles according to $J$ is not lower for farther away landmarks. This confirms the holistic nature of facial perception. The mutual influence among landmarks is not among nearby landmarks only, but over the scale of the entire face.

\subsubsection{Extra information retrieved with effective interactions}

A relevant question is to what extent the inferred effective interactions $J$ provide interpretable information, inaccessible from the raw experimental correlations $C$. In the general case, couples of variables may be statistically correlated through spurious correlations, even in the absence of a causal relation among them (see the Introduction to the Maximum Entropy principle in the SI). In the present case, the main source of spurious correlations is the presence of the constraints among various landmark coordinates. The MaxEnt inference eventually subtracts (through the pseudo-inverse operation) the influence of the constraints from matrix $J$, which describes the essential effective mutual influence among pairs of coordinates of prominent relative importance (see Two ways of inferring with constraints in the database of facial modifications in the SI).

The differences among $C$ and $J$ matrices are apparent from fig. 4-C, where the arrows represent the absolute value of the raw experimental matrix elements $C_{i j}^{\|}$. Remarkably, all but two of the matrix elements result statistically significant $(t$-value $>1)$ : matrix $C^{\|}$can be hardly used to assess the relative relevance of various inter-landmark segments. The effective interaction matrix $J$ disambiguates the correlations propagated by the constraints, attributing them to the effect of a reduced set of elastic constants, in the particle analogy. Such attribution is not unambiguous, but the result of the inference procedure.

In other words, the inferred matrix $J$ provides interpretable results, beyond the less-interpretable empirical information present in matrix $C$. Indeed, matrix $C$ is dense, in the sense that almost all its elements are statistically significant (see fig. 4-C). Matrix $C$ is, however, exactly explained by the 2MaxEnt model, defined by the sparser matrix $J$ of effective interactions, such that only some matrix elements are statistically significant (see figs. 4-A,B).

An in-depth comparison among $C$ and $J$ is presented in the SI, where we consider also the alternative method of avoiding the constraints, consisting in inferring from a non-redundant set of coordinates. We conclude that, in the general case, and for the sake of the interpretation of the effective interactions, it may be convenient to infer from a database of redundant variables, eliminating a posteriori the influence of the null modes associated to the constraints (and, perhaps, of low-variant modes associated to quasi-constraints or to non-linear constraints). 

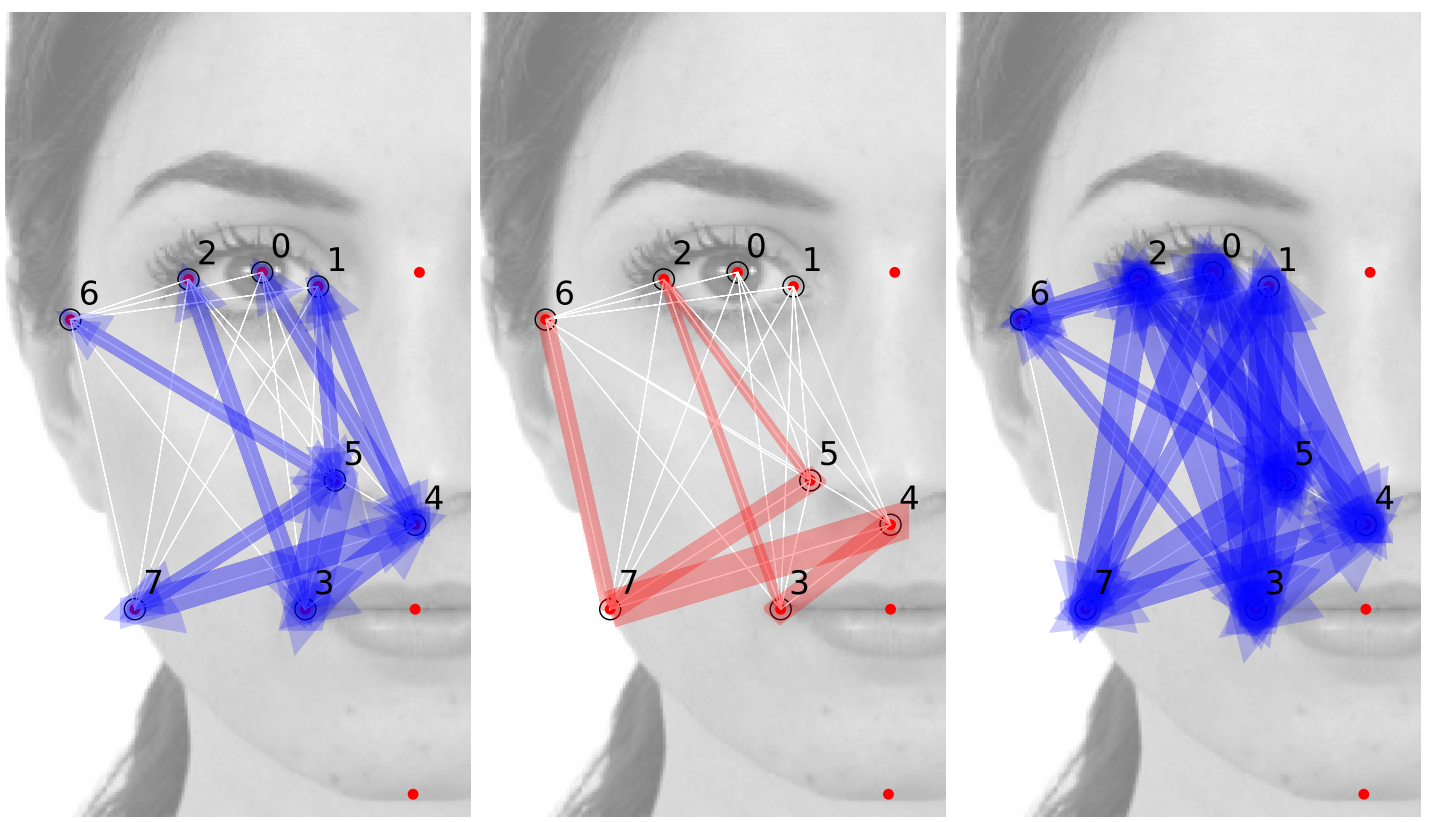

Figure 4. Modulus of the matrices $J^{\|}$(left), $J^{\perp}$ (center), $C^{\|}$(right). The width of the arrow joining the $i$-th and $j$-th landmarks is proportional to $\left|A_{i j}\right|$, where $A$ is the corresponding matrix. Matrices $J^{\|}$and $J^{\perp}$ represent, respectively, the longitudinal and torsion elastic constants of the correspondent inter-landmark segments. They indicate, respectively, the segment's distance and angle "resistance" to differ in the database with respect to their average (preferred) values, reported in the image. Only significant matrix elements have been plotted: only those exhibiting a $t$-value larger than one: $t_{i j}=\left|A_{i j}\right| / \sigma_{A_{i j}}>1$. Matrix $C^{\|}$(right) is less interpretable than $J^{\|}$(left).

\section{CONCLUSIONS}

We have performed an unsupervised inference study of the database of preferred facial modifications presented in reference (Ibáñez-Berganza et al. (2019)). Much work has been devoted to the regression of the average rating in face-space, specially in the machine learning community. Such supervised inference approach indirectly allows for an assessment of the relative impact of various facial traits on perceived attractiveness. This point, however, remains poorly understood (Laurentini and Bottino (2014)). Furthermore, some authors have argued that the subjective nature of facial attractiveness has been overlooked and underestimated, and that the subject-averaged rating presents several limitations as an experimental method (Hönekopp (2006); Laurentini and Bottino (2014); Oh et al. (2019b)). As a novel experimental tool for the investigation of the nature of facial attractiveness and its subjectivity, we here propose an alternative inference scheme in which the variability to be inferred is the inter-subject variability of preferred modifications in a subspace of the face-space (in which only the geometric positions of some landmarks are allowed to vary), rather than the average rating assigned to different natural faces.

The present work is probably the first unsupervised inference approach in facial preference research. Our models induce a probabilistic representations of a set of facial modifications (corresponding to the whole set of subjects or to the set of male or female subjects only, in secs. 3.1.1,3.1.2 respectively, or to the single subject). Such models avoid the use of ratings; account for nonlinear influence of $p$-plets of facial features, hence beyond a principal component analysis; their parameters are in principle interpretable since they involve "physical" facial coordinates only.

Our approach allows to clarify several aspects regarding facial preference. First, that the cognitive mechanisms related to facial discrimination in the brain mainly involves proportions, or pairwise influence of couples of landmarks, more than the positions of single landmarks. Indeed, the 2-MaxEnt model, equivalent to a description in terms of principal components, is enough to describe also non-linear features of the database (see sec. 3.1.1). Moreover, the results suggest as well that non-linear operators of the 
geometrical facial coordinates may play a non-negligible role in the cognitive process (see sec. 3.1.2). Further research is needed to clarify this point (see also Cognitive origin of non-linear correlations in the SI). ${ }^{8}$

Second, and rather remarkably, the introduction of non-linear effective interactions, beyond the influence of proportions, allows for an astonishingly high classification efficiency of the facial vectors according the subject's gender. Indeed, the random forest algorithm, a highly nonlinear supervised algorithm for classification, provides a $97 \%$ of correct classifications. The most non-linear of the probabilistic models, the GRBM, provides a slightly lower accuracy: 95\%. This implies that the subject's gender strikingly determines her/his facial preference criteria and that the sculpted facial modifications, as a sample of the subjects idiosyncratic criterion, are accurate enough to allow to predict such impact.

The impact of the gender is consistent with the sexual selection hypothesis (Little et al. (2011); Rhodes (2006); Thornhill and Gangestad (1999)). However, since the sculpted vectors partially capture the subjects' idiosyncrasy, such a result is also consistent with the multiple motive hypothesis, assuming that the gender strongly influences the subjects' idiosyncratic preferences for personality traits (in the language of Oh et al. (2019b)).

In summary, we have presented probabilistic generative models of the database of preferred facial variations, describing the inter-subject fluctuations around the average modification (given a reference background portrait). The simplest of these models, characterised by pairwise correlations among facial distances, already provides a faithful description of the database. Afterwords, we demonstrate that such fluctuations encode, and may accurately reveal when introducing non-linearity, the subjects' gender. According to the multiple motive hypothesis, many other subject attributes and distinguishing psychological traits may influence, beyond the gender, the preferences in the face-space. The present results suggest that such attributes could be retrieved from the subject sculpted facial vectors.

Finally, we have demonstrated that the data elicited with the method in (Ibáñez-Berganza et al. (2019)) represents a novel case of study for the application of statistical learning methods, in particular the assessment of the relevant order of interaction by comparison with an ANN model, and the comparison among various strategies of inference in the presence of constraints.

The introduction of texture degrees of freedom in the sculpting process is a possible development of the empirical technique of Ibáñez-Berganza et al. (2019), that would allow to quantify the extent to which texture and geometric facial features (and which ones) influence each other in attractiveness perception (a debated question, see Laurentini and Bottino (2014)). Further possible extensions are: the generalisation to different datasets and facial codification methods allowing, for example, landmark asymmetry (see also Generality of the unsupervised inference models in the SI); the classification of different subject's features from her/his set of sculpted faces.

\section{ACKNOWLEDGEMENTS}

We acknowledge Andrea Gabrielli, Irene Giardina, Carlo Lucibello, Giorgio Parisi and Massimiliano Viale for inspiring discussions. Particular thanks to Andrea Cavagna for his suggestions and comments to the draft.

\section{REFERENCES}

Abir, Y., Sklar, A. Y., Dotsch, R., Todorov, A., and Hassin, R. R. (2017). The determinants of consciousness of human faces. Nature Human Behaviour, page 1.

Adolphs, R., Nummenmaa, L., Todorov, A., and Haxby, J. V. (2016). Data-driven approaches in the investigation of social perception. Phil. Trans. R. Soc. B, 371(1693):20150367.

Balas, B. and Pacella, J. (2015). Artificial faces are harder to remember. Computers in human behavior, 52:331-337.

Berg, J. (2017). Statistical mechanics of the inverse ising problem and the optimal objective function. Journal of Statistical Mechanics: Theory and Experiment, 2017(8):083402.

Bialek, W., Cavagna, A., Giardina, I., Mora, T., Silvestri, E., Viale, M., and Walczak, A. M. (2012). Statistical mechanics for natural flocks of birds. Proceedings of the National Academy of Sciences, 109(13):4786-4791.

\footnotetext{
${ }^{8}$ The recent de-codification of the neural code for facial recognition in the primate brain (Chang and Tsao (2017)) has revealed that recognition is based on linear operations (or projections in the geometric and texture principal axes) in the face-space.
} 
Bialek, W. and Ranganathan, R. (2007). Rediscovering the power of pairwise interactions. arXiv preprint arXiv:0712.4397.

Bzdok, D., Langner, R., Caspers, S., Kurth, F., Habel, U., Zilles, K., Laird, A., and Eickhoff, S. B. (2011). ALE meta-analysis on facial judgments of trustworthiness and attractiveness. Brain Structure and Function, 215(3-4):209-223.

Chang, L. and Tsao, D. Y. (2017). The code for facial identity in the primate brain. Cell, 169(6):10131028.e14.

Cossu, G., Del Debbio, L., Giani, T., Khamseh, A., and Wilson, M. (2019). Machine learning determination of dynamical parameters: The ising model case. Phys. Rev. B, 100:064304.

Cunningham, M., Roberts, A., Barbee, A., Druen, P., and Wu, C.-H. (1995). Their ideas of beauty are, on the whole, the same as ours. Journal of Personality and Social Psychology, 68.

Edler, R. J. (2001). Background considerations to facial aesthetics. Journal of Orthodontics, 28(2):159168.

Eisenthal, Y., Dror, G., and Ruppin, E. (2006). Facial attractiveness: Beauty and the machine. Neural Computation, 18(1):119-142. PMID: 16354383.

Galantucci, L. M., Gioia, E. D., Lavecchia, F., and Percoco, G. (2014). Is principal component analysis an effective tool to predict face attractiveness? a contribution based on real $3 \mathrm{~d}$ faces of highly selected attractive women, scanned with stereophotogrammetry. Medical \& Biological Engineering \& Computing, 52(5):475-489.

Hahn, A. C. and Perrett, D. I. (2014). Neural and behavioral responses to attractiveness in adult and infant faces. Neuroscience \& Biobehavioral Reviews, 46:591-603.

Higgins, I., Chang, L., Langston, V., Hassabis, D., Summerfield, C., Tsao, D., and Botvinick, M. (2020). Unsupervised deep learning identifies semantic disentanglement in single inferotemporal neurons.

Hönekopp, J. (2006). Once more: is beauty in the eye of the beholder? relative contributions of private and shared taste to judgments of facial attractiveness. Journal of Experimental Psychology: Human Perception and Performance, 32(2):199.

Ibáñez-Berganza, M., Amico, A., and Loreto, V. (2019). Subjectivity and complexity of facial attractiveness. Scientific Reports, 9:8364.

Jaynes, E. T. (1957). Information theory and statistical mechanics. Phys. Rev., 106:620-630.

Laurentini, A. and Bottino, A. (2014). Computer analysis of face beauty: A survey. Computer Vision and Image Understanding, 125:184-199.

Leopold, D. A. and Rhodes, G. (2010). A Comparative View of Face Perception. Journal of comparative psychology (Washington, D.C. : 1983), 124(3):233-251.

Lezon, T. R., Banavar, J. R., Cieplak, M., Maritan, A., and Fedoroff, N. V. (2006). Using the principle of entropy maximization to infer genetic interaction networks from gene expression patterns. Proceedings of the National Academy of Sciences, 103(50):19033-19038.

Little, A., Jones, B., and DeBruine, L. (2011). Facial attractiveness: evolutionary based research. Philosophical Transactions, (366).

Little, A. C. (2014). Facial attractiveness. Wiley Interdisciplinary Reviews: Cognitive Science, 5(6):621634.

Ma, D. S., Correll, J., and Wittenbrink, B. (2015). The Chicago face database: A free stimulus set of faces and norming data. Behavior Research Methods, 47(4):1122-1135.

MacKay, D. J. (2003). Information theory, inference and learning algorithms. Cambridge university press.

Martino, A. D. and Martino, D. D. (2018). An introduction to the maximum entropy approach and its application to inference problems in biology. Heliyon, 4(4):e00596.

Melchior, J. (2017). Pydeep. https: / / github. com/MelJan/PyDeep.git.

Monechi, B., Ibáñez-Berganza, M., and Loreto, V. (2020). Hamiltonian modeling of macro-economic urban dynamics. Royal Society Open Science (in press).

Mora, T., Walczak, A. M., Bialek, W., and Callan, C. G. (2010). Maximum entropy models for antibody diversity. Proceedings of the National Academy of Sciences, 107(12):5405-5410.

Morcos, F., Pagnani, A., Lunt, B., Bertolino, A., Marks, D. S., Sander, C., Zecchina, R., Onuchic, J. N., Hwa, T., and Weigt, M. (2011). Direct-coupling analysis of residue coevolution captures native contacts across many protein families. Proceedings of the National Academy of Sciences, 108(49):E1293-E1301. Murphy, K. (2012). Machine Learning: A Probabilistic Perspective. The MIT Press. 
Nguyen, H. C., Zecchina, R., and Berg, J. (2017). Inverse statistical problems: from the inverse ising problem to data science. Advances in Physics, 66(3):197-261.

Oh, D., Dotsch, R., and Todorov, A. (2019a). Contributions of shape and reflectance information to social judgments from faces. Vision research, 165:131-142.

Oh, D., Grant-Villegas, N., and Todorov, A. (2019b). The eye wants what the heart wants: Female face preferences track partner personality preferences.

Oosterhof, N. N. and Todorov, A. (2008). The functional basis of face evaluation. Proceedings of the National Academy of Sciences, 105(32):11087-11092.

Rhodes, G. (2006). The Evolutionary Psychology of Facial Beauty. Annual Review of Psychology, 57(1):199-226.

Roudi, Y., Aurell, E., and Hertz, J. A. (2009). Statistical physics of pairwise probability models. Frontiers in computational neuroscience, 3:22.

Schneidman, E., Berry II, M. J., Segev, R., and Bialek, W. (2006). Weak pairwise correlations imply strongly correlated network states in a neural population. Nature, 440(7087):1007.

Shlens, J., Field, G. D., Gauthier, J. L., Grivich, M. I., Petrusca, D., Sher, A., Litke, A. M., and Chichilnisky, E. (2006). The structure of multi-neuron firing patterns in primate retina. Journal of Neuroscience, 26(32):8254-8266.

Stephens, G. J. and Bialek, W. (2010). Statistical mechanics of letters in words. Physical Review E, 81(6):066119.

Tang, A., Jackson, D., Hobbs, J., Chen, W., Smith, J. L., Patel, H., Prieto, A., Petrusca, D., Grivich, M. I., Sher, A., et al. (2008). A maximum entropy model applied to spatial and temporal correlations from cortical networks in vitro. Journal of Neuroscience, 28(2):505-518.

Thornhill, R. and Gangestad, S. W. (1999). Facial attractiveness. Trends in Cognitive Sciences, 3(12):452 -460 .

Tkacik, G., Schneidman, E., Berry, I., Michael, J., and Bialek, W. (2009). Spin glass models for a network of real neurons. arXiv preprint arXiv:0912.5409.

Todorov, A. and Oosterhof, N. N. (2011). Modeling Social Perception of Faces [Social Sciences]. IEEE Signal Processing Magazine, 28(2):117-122.

Trigueros, D. S., Meng, L., and Hartnett, M. (2018). Face recognition: From traditional to deep learning methods. arXiv preprint arXiv:1811.00116.

Valentine, T., Lewis, M. B., and Hills, P. J. (2016). Face-space: A unifying concept in face recognition research. The Quarterly Journal of Experimental Psychology, 69(10):1996-2019.

Walker, M. and Vetter, T. (2016). Changing the personality of a face: Perceived Big Two and Big Five personality factors modeled in real photographs. Journal of personality and social psychology, 110(4):609.

Wang, N., Melchior, J., and Wiskott, L. (2012). An analysis of gaussian-binary restricted boltzmann machines for natural images. In Proc. 20th European Symposium on Artificial Neural Networks, Computational Intelligence and Machine Learning, Apr 25-27, Bruges, Belgium, page 287-292.

Wang, N., Melchior, J., and Wiskott, L. (2014). Gaussian-binary restricted boltzmann machines on modeling natural image statistics. CoRR, abs/1401.5900.

Weigt, M., White, R. A., Szurmant, H., Hoch, J. A., and Hwa, T. (2009). Identification of direct residue contacts in protein-protein interaction by message passing. Proceedings of the National Academy of Sciences, 106(1):67-72.

Xu, J., Jin, L., Liang, L., Feng, Z., Xie, D., and Mao, H. (2017). Facial attractiveness prediction using psychologically inspired convolutional neural network (PI-CNN). In 2017 IEEE International Conference on Acoustics, Speech and Signal Processing (ICASSP), pages 1657-1661. 